\title{
Monte Carlo Investigation of Spatially Adaptable Magnetic Behavior in Stretchable Uniaxial Ferromagnetic Monolayer Film
}

\author{
Yongyut Laosiritaworn $^{1 *}$ and Wimalin Laosiritaworn ${ }^{2}$ \\ ${ }^{1}$ Department of Physics and Materials Science, Faculty of Science, Chiang Mai University, Chiang Mai 50200, Thailand \\ ${ }^{2}$ Department of Industrial Engineering, Faculty of Engineering, Chiang Mai University, Chiang Mai 50200, Thailand
}

(Received 5 July 2014, Received in final form 23 January 2015, Accepted 6 February 2015)

\begin{abstract}
In this work, Monte Carlo simulation was employed to model the stretchable Ising monolayer film to investigate the effect of the spatial distance variation among magnetic atoms on magnetic behavior of the film. The exchange interaction was considered as functions of initial interatomic distance and the stretched distance (or the strain). Following Bethe-Slater picture, the magnetic exchange interaction took the Lennard-Jones potential-like function. Monte Carlo simulations via the Wolff and Metropolis algorithms were used to update the spin systems, where equilibrium and dynamic magnetic profiles were collected. From the results, the strain was found to have strong influences on magnetic behavior, especially the critical behavior. Specifically, the phase transition point was found to either increase or decrease depending on how the exchange interaction shifts (i.e. towards or away from the maximum value). In addition, empirical functions which predict how the critical temperatures scale with initial interatomic distance and the strain were proposed, which provides qualitatively view how to fine tune the magnetic critical point in monolayer film using the substrate modification induced strain.
\end{abstract}

Keywords : ising model, magnetic behavior, monte carlo simulation, stretchable film

\section{Introduction}

Ferromagnetic materials in very thin film structure have long been accepted as key materials for recording digital data in terms of data accessibility and low cost (capacity per unit price) $[1,2]$. Furthermore, especially in this information technology driven era, the majority of accessing data, ranging from movie industries to medical research, are now kept in digital format for the benefit of data transferring and real time access. This therefore stresses out the importance and the need for enhancing the capacity of the ferromagnetic film beyond current obstructing limit, where both theoretical and experimental lines of research have to be comprehensively cooperated. Nevertheless, due to the structural complexity in low (reduced) dimension, theoretical studies on magnetic materials cannot be trivially taken. For instance, many previous studies consider the ferromagnetic film in somewhat separating parts from the device. However, in real application, the

CThe Korean Magnetics Society. All rights reserved.

*Corresponding author: Tel: +66-53-94-3367

Fax: +66-53-94-3445, e-mail: yongyut_laosiritaworn@yahoo.com film is usually grown the substrate, coupled with different materials layers, or attached to the electrode. In general, when the film is very thin, the interatomic distance (lattice spacing between spins) was stretched to match with that of its coupling layers. Note that, when there is some lattice spacing mismatch between the ferromagnetic film and its environment, there exist intrinsic stress and strain propagating throughout the film. Then, this intrinsic stress and strain (with large enough magnitude) can induce structural anisotropies $[3,4]$ and cause the magnetic behavior to be deviated from the ideal stress-strain free condition. For instance, in some magnetic systems, the anisotropies arisen from stresses were found to essentially shift the magnetic phase transition and its critical phenomena [5-8]. As a result, it is very important to consider the stretchable phenomena and its corresponding latticemismatch induced strain in modelling real ferromagnetic materials especially in thin-film structure (reduced geometry), where the magnetostrictive effect (or the magnetostriction) is very important.

The magnetostriction effect is a property of ferromagnetic materials where the materials change their dimensions during the alteration of magnetization. To explain, the 
magnetostriction is usually termed with a magnetostrictive coefficient, defined as the fractional change in dimension as the magnetization changes from zero to its maximum magnitude. On the other hand, the applied mechanical strain can also produce some magnetic anisotropy. In such the case, the direction of the applied stress can become the preferred ferromagnetic direction. Note that magnetization can be rotated by the applied magnetic field via the magnetic work. Consequently, if the stress induced magnetization preferred direction is different from the applied magnetic field direction, there could be some substantial change in the ferromagnetic properties according to these mechanical and magnetic competitive effects on the domain wall movements. These magnetostriction phenomena have long been discovered and evident in investigations. For instance, a recent study on anisotropies in Co films grown on $\mathrm{Mo}(110)$ confirms the magnetostriction [9]. Various kinds of anisotropies (in-plane, outof-plane, interface and surface anisotropy) as well as its origin have been investigated and reported. There was anisotropy contributed from epitaxial strain in addition to the bulk crystal anisotropy, which confirms that strain can induce magnetic anisotropies. Another example was on Fe grown as step decoration on a curved $\operatorname{Pt}(111)$ single crystal [10]. It was found that the step-induced magnetic anisotropy enhances the Curie temperature of $\mathrm{Fe}$ ultrathin films, and the step-induced surface anisotropy was found to be proportional to step's vicinal angle. Theoretical works also validate the magnetostriction arguments. A study on GdN reported that, by using first principles based calculation, the strain can induce the half-metal to semiconductor transition [11]. In brief, there was a transformation in the conduction properties associated with the volume increase (half-metal to semiconductor). The exchange parameters were also found to strongly depend on lattice constant, which implies Curie temperature dependence of the applying stress.

Furthermore, it was found that ferromagnetic and antiferromagnet preferences are interchangeable depending on the magnitude and type of the stress $[5,7,12]$. In addition, the compressible Ising spin model was investigated where the paramagnetic volume expansion was found to depend on the strength of the magnetoelastic coupling [13], while the critical temperature was found to vary as a result of changing applied stresses [14-20]. In terms of dynamic behavior, the hysteresis remanence reducing with increasing the compressive stress was reported [21]. Nevertheless, previous theoretical investigations usually considered the effect of stresses in allowing the magnetic atoms to move around their equilibrium position which alters the magnetic interaction among spins and overall magnetic properties. On the other hand, how both stress induced lattice distortion from its original position and how its magnitude conclusively play their vital role on magnetic properties has not been investigated in details, e.g. the universal function which can predict how much the critical temperature varies as changing strain and the original lattice spacing is still yet to identify.

Therefore, as a part in filling the gap, this work aims to provide another step in understanding/advancing the ferromagnetic thin-film topic to answer the magnetic behavior and its critical temperature changing problem in real situation. The lattice distortion (strain) and the original lattice spacing (unstrained interatomic distance) were incorporated into the magnetic system via the space dependent magnetic exchange interaction. The Ising Hamiltonian was considered as it does suit strong crystalline magnetic anisotropies in film structure. The exchange interaction was theorized to be quantified by the LennardJones (LJ) function as its qualitative characteristic is in line with the Bethe-Slater picture. Next, the ferromagnetic spins were updated using Monte Carlo simulation in extracting magnetic properties. Finally, the results were analyzed to extract magnetic profiles and the critical temperature as functions of strain and initial (original) interatomic distance, while empirical functions in predicting the critical temperature have been successfully found. The major outcome of this work will then be of some benefit to technologists on how to fine tuning the ferromagnetic phase transition by choosing appropriate substrate.

\section{Stretchable Ising Hamiltonian and Monte Carlo Procedure}

In this work, the ferromagnetic spin model is the uniaxial infinite anisotropic Ising model on 2-dimensional square lattice, i.e. ultra-thin-film. Note that in bulk structure, the Heisenberg model can be a choice of consideration as it is equipped with full rotational symmetric in the spatial space. However, the 2-dimensional classical isotropic Heisenberg model (even the spin can point into any directions in the spatial space), it has been rigorously proved that the system without long ranged interactions and under the absence of external field has no ferromagnetic ordering at finite temperatures [22,23]. Specifically, the magnetization is zero at all nonzero temperatures i.e.

$$
\left\langle m_{z}\right\rangle_{N \rightarrow \infty} \propto \frac{1}{\sqrt{T\left|\ln h_{z}\right|}} \rightarrow 0 \text { as } h_{z} \rightarrow 0,
$$

where $h_{z}$ is the magnitude of the external field applied 
along $z$ direction. A similar theorem was also proposed to confirm that the symmetry breaking field is required for any finite temperatures to yield ferromagnetic ordering at thermodynamic limit $(N \rightarrow \infty)$ i.e. [24]

$$
\left\langle m_{z}^{2}\right\rangle_{N \rightarrow \infty} \propto \frac{1}{\ln N} \rightarrow 0 \text { as } N \rightarrow \infty
$$

Therefore to model ferromagnetic ordering, strong anisotropies are required. This can be modeled by introducing anisotropies in the exchange interaction energy i.e. [25]

$$
H=-J \sum_{\langle i j\rangle}\left[s_{i z} \cdot s_{j z}+(1-\Delta)\left(s_{i x} s_{j x}+s_{i y} s_{j y}\right)\right]
$$

where $\hat{s}_{i}=\left(s_{i x}, s_{i y}, s_{i z}\right)$ is the unit-vector classical Heisenberg spin at site $i$, and $\Delta$ is the uniaxial exchange anisotropy. Note that with varying $\Delta$ from 0 to 1 , the system changes from typical classical Heisenberg spin to the Ising-like spin model, where the phase transition of ferromagnetic onset shifts to zero temperature for $\Delta$ approaching 0 .

Also, it is well-known that uniaxial anisotropies are very strong in films' structure. The Ising model, which is an infinite uniaxial anisotropic model, then becomes suitable for investigating ferromagnetic thin-films. This Ising model has been proved useful where many theoretical $[26,27]$ and experimental works [28-30] have confirmed that the magnetic behavior in thin-films structure has the Ising universality. Consequently, the Ising model was then considered in this work to investigate the ferromagnetic ordering and its critical behavior in ultra-thin-film structure. Being stretchable, the considered Ising Hamiltonian

(a)

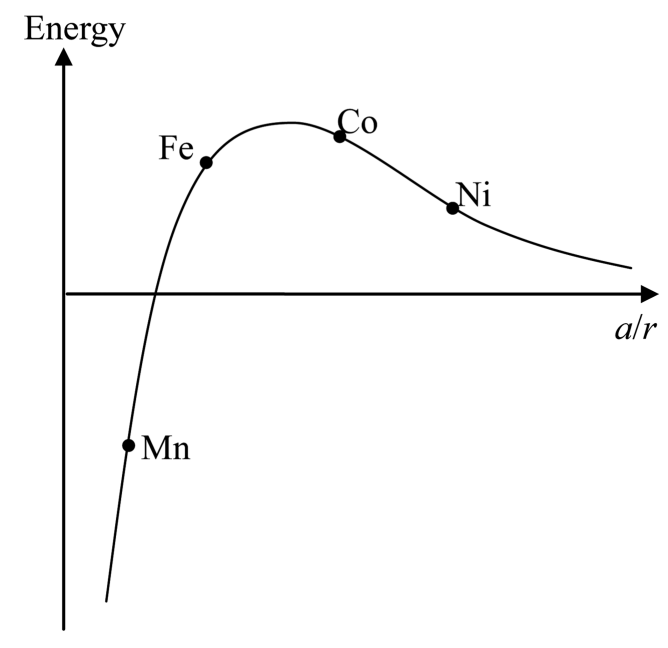

for describing the magnetic interaction in the stretchable system then take the form i.e.

$$
H=-\sum_{\langle i j\rangle} J_{i j} s_{i} s_{j}-h(t) \sum_{i} s_{i},
$$

where the spin $s_{i}= \pm 1$ referring to the spin's direction, the notation $<i j>$ indicates the sum includes only nearest neighboring pairs, $J_{i j}$ is the spatial dependent exchange interaction among spins which is a function of the distance between spins $s_{i}$ and $s_{i}$ (the interatomic distance or the lattice spacing), and $h(t)=h_{0} \sin (2 \pi f t)$ where $h_{0}$ is the field amplitude and $f$ is the field frequency. In Eq. (1), as $J_{i j}$ is a function of distance between spins $s_{i}$ and $s_{j}$, its magnitude becomes the function of the existing strain in the system. In general, the exchange interaction is a result of exchanged wave function overlapping among electrons. Therefore, with changing the lattice spacing, the exchange interaction will change in its value leading to some change in the overall magnetic behavior, e.g. the interchanging between ferromagnetic and anti-ferromagnetic preferences $[5,7,12]$. One descriptive picture which explains the change of exchange interaction magnetic with distance was given by the Bethe-Slater curve, e.g. see Fig. 1(a). As can be seen, the Bethe-Later curve schematically tells what magnetic element is ferromagnetic and what magnetic element is anti-ferromagnetic. In addition, it is clearly seen that if the interatomic distance is slightly changed while the radius of the $3 d$ electron shell does not change much, it is possible to tune the magnetic behavior with changing the interatomic distance. However, the true formalism for the Bethe-Slater function is still yet to be

(b)

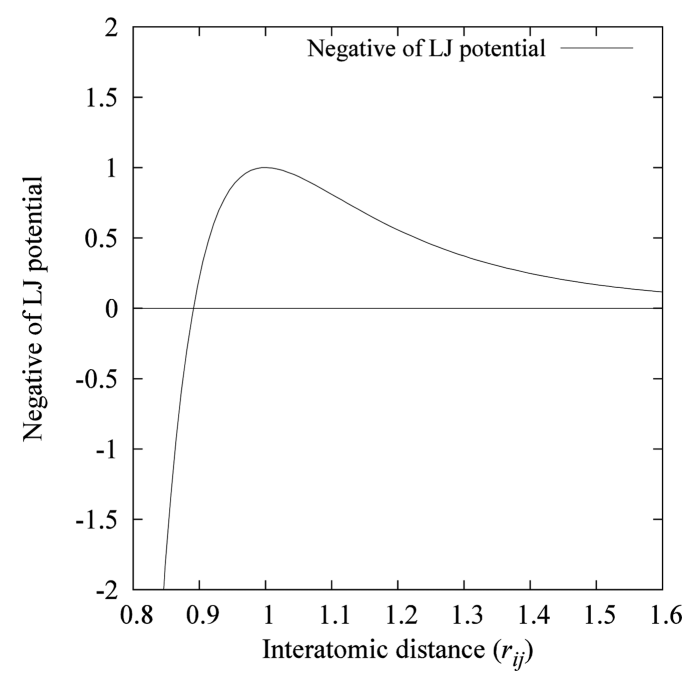

Fig. 1. (a) The typical schematic Bethe-Slater diagram displaying exchange interaction energy of magnetic materials as a function of the interatomic spacing to the radius of the $3 \mathrm{~d}$ electron shell ratio $(a / r)$ in comparison with (b) the negative value of the LennardJones (LJ) potential. 
exactly defined. Therefore, in this work, the exchange interaction function was assumed to take a negative form of the Lennard-Jones potential-like function i.e. [31-33]

$$
J_{i j}=-J_{0}\left[\left(\frac{\sigma}{r_{i j}}\right)^{12}-2\left(\frac{\sigma}{r_{i j}}\right)^{6}\right] \text {, }
$$

where with varying the interatomic distance $r_{i j}$ between spins $i$ and $j$, the change in $J_{i j}$ can be displayed as in Fig. 1(b). In Eq. (2), $\sigma$ refers to the interatomic distance that yield maximum value of the Lennard-Jones potential, and $J_{0}$ is the maximum ferromagnetic exchange interaction associated to $\sigma$. Then, with changing the unit of distance to $\sigma, \sigma$ can be set to 1 while unit of $r_{i j}$ was redefined as $\sigma$.

Therefore, in this work, the effect of the anisotropy due to the strain was considered and prescribed by the LennardJones type exchange interaction among spins, i.e. Eq. (2). This is as when finite stress is applied onto the system, the strain is induced (via modulus of elasticity) and the lattice spacing between each pair of magnetic atoms repositions in responding to the applied mechanical work. With the change in this lattice spacing and the use of Eq. (2), the anisotropies in the exchange interaction were introducing among spins. Note that the Ising spin has only one component, e.g. in the $z$-direction. Therefore, the Ising is in fact anisotropic model. Therefore, the stress/ strain inducing anisotropies in this work is actually to strengthen or to weaken anisotropic interaction magnitude among the Ising spins.

More specifically, the stress/strain inducing anisotropies were employed only in the context of spin-dimension, not the spatial-dimension. This prescription is applicable as the stress considered in this work has a form of hydrostatic pressure applying on the system with the same magnitude in all directions. Consequently, the symmetry breaking field is not intrinsically induced in a particular direction due to this $4 \pi$ symmetry. Therefore, the anisotropic direction of the Ising spins does not change. Note that the change of magnetic exchange interaction due to the stress/ strain (which results in the change ferromagnetic ordering and its phase transition) has been long pointed out in experiments $[34,35]$, where in this work this effect was taken into account as a function of spin-spin distances.

In addition, from Eq. (2), $J$ may become negative (antiferromagnetic preference) for small $r_{i j}$ e.g. for $r_{i j}<1 / \sqrt[6]{2}$ $\approx 0.8909$. This is outside the scope of ferromagnetic behavior investigation in this work, so the initial (original) interatomic distance $r_{\text {init }}$ and the strain $\Delta r_{i j} / r_{\text {init }}$ between the neighboring pairs of spins have to be carefully chosen. For some common ferromagnetic materials, the value of the $r_{\text {init }}$ without the $\sigma$ normalization are presented [36, 37], as shown in Table 1.

Also, when growing films on a substrate, the lattice spacing between the film and the substrate should not be very much different unless the film may not attach on. Consequently, this work considered the range of the strain, which is the ratio of lattice distortion to its original size, being less than 5 percent. In this work, the interatomic distance $r_{\text {init }}$ ranged from 0.96 to 1.04 , whereas the strain $\Delta r_{i j} / r_{\text {init }}$ ranged from -0.05 to 0.05 . Consequently, the resultant interatomic spacing $r_{i j}$ and the negative value of

Table 1. Atomic separation, the unfilled $3 d$ electron radius and their ratios for elements $\mathrm{Fe}$, Co and $\mathrm{Ni}$.

\begin{tabular}{cccccc}
\hline $\begin{array}{c}\text { Ferromagnetic } \\
\text { materials }\end{array}$ & $\begin{array}{c}\text { Crystal } \\
\text { structure }\end{array}$ & $\begin{array}{c}\text { Atomic separation } \\
a(\mathrm{~nm})[37]\end{array}$ & $\begin{array}{c}\text { unfilled } 3 d \text { electron } \\
\text { shell radius } r(\mathrm{~nm})[37]\end{array}$ & $\begin{array}{c}\text { Curie temperature } \\
(\mathrm{K})[36]\end{array}$ \\
\hline $\mathrm{Fe}$ & $\mathrm{BCC}$ & 0.250 & 0.0790 & 3.26 & 1044 \\
$\mathrm{Co}$ & FCC & 0.251 & 0.0690 & 3.64 & 1388 \\
$\mathrm{Ni}$ & FCC & 0.250 & 0.0635 & 3.94 & 628 \\
\hline
\end{tabular}

Table 2. The interatomic distance (or lattice spacing) and negative values of the LJ potential for various initial spacing $r_{\text {init }}$ and strain $\Delta r_{i j} / r_{\text {init }}$.

\begin{tabular}{|c|c|c|c|c|c|c|c|c|c|c|}
\hline \multirow{2}{*}{$\begin{array}{l}\text { Strain } \\
\Delta r_{i j} / r_{\text {init }}\end{array}$} & \multicolumn{5}{|c|}{ Interatomic distance $r_{i j}$ (with varying $\left.r_{i n i t}\right)$} & \multicolumn{5}{|c|}{$\begin{array}{l}\text { Negative of LJ potential or the exchange interaction } J_{i j} \\
\text { (with varying } r_{\text {init }} \text { ) }\end{array}$} \\
\hline & 0.96 & 0.98 & 1.00 & 1.02 & 1.04 & 0.96 & 0.98 & 1.00 & 1.02 & 1.04 \\
\hline-0.05 & 0.9120 & 0.9310 & 0.9500 & 0.9690 & 0.9880 & 0.4555 & 0.7130 & 0.8701 & 0.9567 & 0.9944 \\
\hline-0.02 & 0.9408 & 0.9604 & 0.9800 & 0.9996 & 1.0192 & 0.8045 & 0.9247 & 0.9834 & 1.0000 & 0.9884 \\
\hline-0.01 & 0.9504 & 0.9702 & 0.9900 & 1.0098 & 1.0296 & 0.8726 & 0.9604 & 0.9961 & 0.9968 & 0.9742 \\
\hline 0.00 & 0.9600 & 0.9800 & 1.0000 & 1.0200 & 1.0400 & 0.9230 & 0.9834 & 1.0000 & 0.9874 & 0.9560 \\
\hline 0.01 & 0.9696 & 0.9898 & 1.0100 & 1.0302 & 1.0504 & 0.9586 & 0.9960 & 0.9966 & 0.9733 & 0.9347 \\
\hline 0.02 & 0.9792 & 0.9996 & 1.0200 & 1.0404 & 1.0608 & 0.9819 & 1.0000 & 0.9874 & 0.9553 & 0.9111 \\
\hline 0.05 & 1.0080 & 1.0290 & 1.0500 & 1.0710 & 1.0920 & 0.9978 & 0.9752 & 0.9356 & 0.8862 & 0.8317 \\
\hline
\end{tabular}


the LJ potential (the distance dependent exchange interaction) can be shown as in Table 2 .

To summarize parameters setting up, $J_{0}=1$ was used as the energy unit so $J_{0} / k_{B}$ (where $k_{B}$ is the Boltzmann's constant) was the unit of temperature. In the static simulation, i.e. without the applied magnetic field, the simulations were carried out for a range of temperatures $T$ $=0.05$ to $3.00 J_{0} / k_{B}$. However, for the dynamic simulation where the magnetic field was turned on, the temperature $T$ was ranged from 1.00 to $2.60 J_{0} / k_{B}$, the field amplitude $h_{0}$ was ranged from 0.25 to $4.00 J_{0}$, but the field frequency $f$ was fixed only at $f=0.01 \mathrm{mcs}^{-1}$ which was found to balance between computational time and welldefined hysteresis loops. The initial interatomic distance $r_{\text {init }}$ and strain $\Delta r_{i j} / r_{\text {init }}$ parameters were set as in Table 2. The considered system was simulated on the two-dimensional square lattice (with periodic boundary condition on all sides). Therefore, the total number of spins was $N=$ $L^{2}$, where $L$ ranged from 20 to 90 (in a step of 10). Note that, the difference in magnetic behavior is not significantly found for larger $L$, except the critical point where finite size scaling technique was used to overcome the problem. The unit time was defined from one full simulation update of all spins, i.e. 1 Monte Carlo step per site (mcs).

This work firstly investigated the static ferromagnetic magnetic behavior of the system by setting $h(t)=0$ at all time $t$. Then, in updating the spin configurations, the Wolff algorithm [38] was used to minimize the correlation time and statistical errors [39]. During the configuration updating, a cluster of spins were made by adding same spins with a probability

$$
p_{i j}=1-\exp \left\{\min \left(0,-\frac{2 J_{i j} s_{i} s_{j}}{k_{B} T}\right)\right\} .
$$

After that, all spins in the same cluster were flipped to their opposite directions i.e. $\left\{s_{i}\right\}$ to $\left\{-s_{i}\right\}$. For equilibration, each simulation was firstly waited for $1,000 \mathrm{mcs}$, and then the magnetization and the energy were measured when the number of flipped spins exceeding or being equal to $N$. At least $t_{\max }=5,000$ different configurations were used to calculate the expectation of the magnetization per spin

$$
\langle m\rangle=\frac{1}{t_{\max }} \sum_{t=0}^{t_{\max }}\left|m_{i}\right|\left(\text { where } m=\frac{1}{N} \sum_{i=1}^{N} s_{i}\right)
$$

as well as the magnetic susceptibility

$$
\chi=\frac{N}{k_{B} T}\left(\left\langle m^{2}\right\rangle-\left\langle m^{2}\right\rangle\right) \text {. }
$$

Next, the critical temperature $T_{C}$ was extracted using the fourth order cumulant of the magnetization per spin $U_{L}$ i.e. [40]

$$
U_{L}=1-\frac{\left\langle m^{4}\right\rangle}{3\left\langle m^{2}\right\rangle^{2}},
$$

where at critical temperature the cumulant $U_{L}$ becomes $L$ independent i.e. $\left(U_{L} / U_{L^{\prime}}\right)_{T=T_{C}}=1$ for differing sized $L$ and $L^{\prime}$. However, due to finite size effects, the cumulant for different $L$ 's may not cross at a same temperature, so $T_{C}$ should be estimated from $T_{C}\left(b=L / L^{\prime}\right)$ at the limit $(\ln b)^{-1} \rightarrow 0$ [40]. Also, in optimizing computer resource, only a single long simulation was performed for each $L$ while the single histogram method i.e. [41]

$$
Q_{\text {estimate }}(\beta)=\frac{\Sigma_{E}\langle Q(E)\rangle N(E) \exp \left(-\left(\beta-\beta_{0}\right) E\right)}{\Sigma_{E} N(E) \exp \left(-\left(\beta-\beta_{0}\right) E\right)},
$$

was used to find the cumulant crossing points. In Eq. (7), $Q_{\text {estimate }}$ refers to the estimator of the $<Q>$ at a particular inverse temperature $\beta \equiv 1 / k_{B} T, \beta_{0} \equiv 1 / k_{B} T_{0}, T_{0}$ is the temperature that the long simulation was performed, $E$ is the system energy and $N(E)$ is the histogram at bin $E$. In this work, $10^{6}$ spin configurations were used to construct the histogram as this number was found to compromise between calculation time and statistical error. To locate the critical point, $T_{0}$ should be as close to $T_{C}$ as possible [41], so the temperature where the susceptibility of the $L$ $=90$ system gets maximum was assigned as $T_{0}$ and used for all $L$ 's. Note that for estimating the cumulant $U_{L}$ from single histogram method, the parameters $Q$ 's are $m^{2}$ and $m^{4}$.

Next, for the dynamic simulation, the external magnetic field was applied to the system. The Monte Carlo update algorithm for the spin configuration for this dynamic case used the Metropolis algorithm as the Metropolis update the spin one-by-one so it becomes more practical in use as number of spin flips per field-period has to be the same. The Metropolis probability for updating each individual spin has the form [42]

$$
p_{i j}=\left\{\begin{array}{cc}
\exp \left(-\frac{\Delta E}{k_{B} T}\right) & \text { if } \Delta E>0 \\
1 & \text { otherwise }
\end{array}\right.
$$

where $\Delta E$ is the energy difference between the after- and the before-flip. In each field period, 100 pairs of $(m, h)$ were recorded to form hysteresis loops. The first 1,000 hysteresis loops were discarded for obtaining steady state. Then, the next 5,000 loops were recorded and averaged to obtain the representative of the loop for the considered set 
(a)

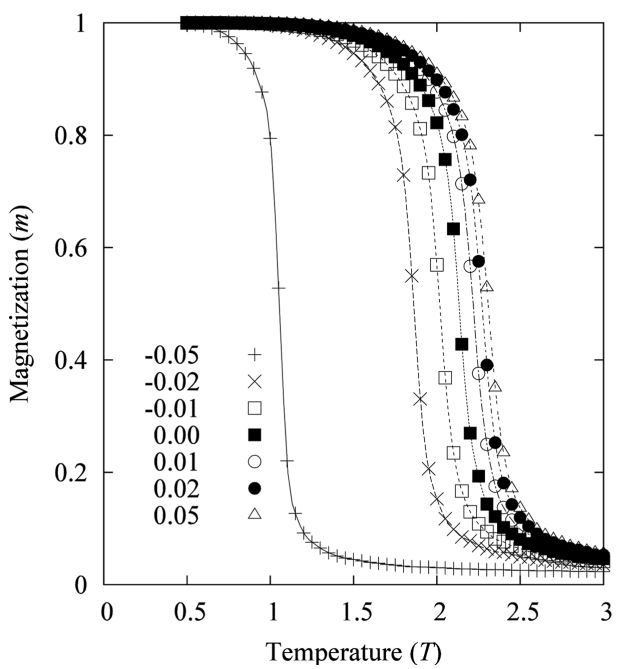

(b)

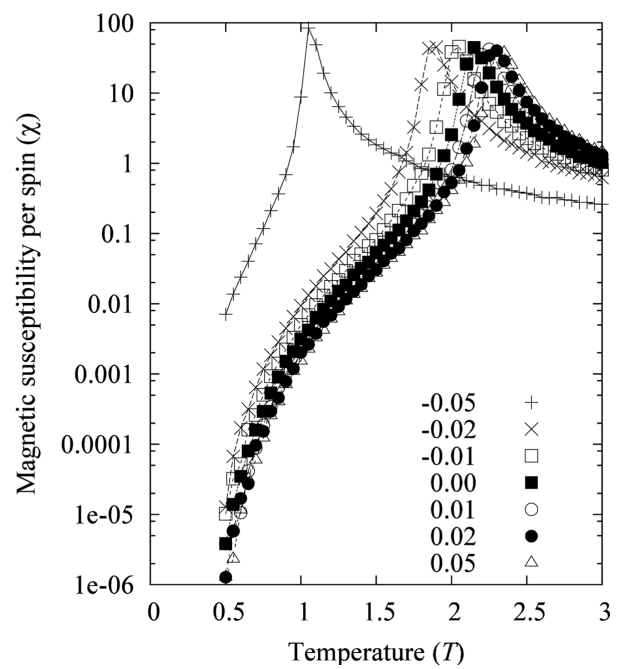

Fig. 2. (a) Magnetization and (b) magnetic susceptibility profiles of the Ising ultra-thin-film having $r_{\text {init }}=0.98$ and the strain $\Delta r_{i j} / r_{\text {init }}$ ranging from -0.05 to 0.05 .

of input parameters.

\section{Results and Discussions}

From the work, the magnetization and magnetic susceptibility profiles were obtained, e.g. see Fig. 2. In the figure, it was found that the phase transition points, where the magnetization curves have the greatest slopes or the susceptibilities result in peaks, tend to shift to higher temperatures when the strain $\Delta r_{i j} / r_{\text {init }}$ increases from -0.05 to 0.05 . This is expected since, as shown in Table 2, for $r_{\text {init }}=0.98$, the exchange interaction monotonically increases with increasing the strain from -0.05 to 0.05 . On the other hand, different $r_{\text {init }}$ does yield different exchange interaction profiles. For instance, at $r_{\text {init }}=1.00$ and with increasing the strain $\Delta r_{i j} / r_{\text {init }}$, the exchange interaction increases and arrives at its maximum value at zero strain (i.e. $\Delta r_{i j} / r_{i n i t}=0$ ) and then declines afterwards. Therefore, Table 2 presents a guideline for predicting the critical point if it should increase or decrease when changing the strain. In addition, Table 2 also provides some description why some magnetic materials have their critical points increased but some do not (e.g. decreased instead) when go under stress loading condition [14-20].

However, although magnetic profiles as shown in Fig. 2 suggest roughly where the critical point should be, this critical point is not the same as that for the system at thermodynamic limit. This is as due to the finite size effect where the correlation length of the spin cluster is limited by the system size causing the magnetic behaviors in finite systems being different from those at the infinite sized system [41]. Therefore, the fourth order cumulant in Eq. (6) was used to extract the critical temperature at thermodynamic limit. As mentioned previously, due to the finite size effect, the cumulant crossing points are not at the same temperature, e.g. see Fig. 3. Therefore, by plotting temperature of the crossing point for pairs of $L$ and $L^{\prime}$, i.e. $T_{C}\left(b=L / L^{\prime}\right)$ where $L>L^{\prime}$, as a function of $\ln ^{-1}(b)$ and performing linear fitting, the critical point can be extracted by extrapolating $b$ to the infinite limit $(b \rightarrow$ $\infty$ or $\ln ^{-1}(b) \rightarrow 0$ ). Since the system sizes in this works ranges from $L=20$ to 90 , three different $L^{\prime}$ which are $L^{\prime}=$

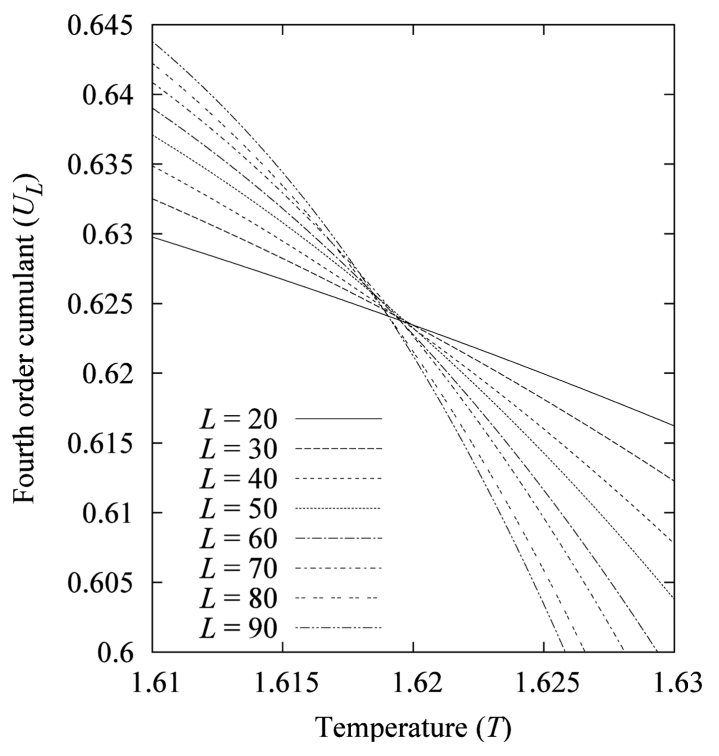

Fig. 3. Results for the cumulant analysis. As is seen, the crossing points for different $L$ 's are not at the same temperature. 
Table 3. Critical temperatures $T_{C}$ extracted using the fourth order cumulant for different initial spacing $r_{\text {init }}$ (upper-half) to the strain ratio, i.e. $\Delta r_{i j} / r_{i n i t}$, and coefficients $\{a, b, c, d\}$ for the degree-3 polynomial function used to fit the $T_{C}$ data (lower-half), e.g. see Fig. 4.

\begin{tabular}{|c|c|c|c|c|c|}
\hline \multirow{2}{*}{$\frac{\text { Strain }}{\Delta r / r_{0}}$} & \multicolumn{5}{|c|}{ Critical temperature $T_{C}$ (varying $\left.r_{\text {init }}\right)$} \\
\hline & $r_{i n i t}=0.96$ & $r_{i n i t}=0.98$ & $r_{i n i t}=1.00$ & $r_{\text {init }}=1.02$ & $r_{\text {init }}=1.04$ \\
\hline-0.05 & $1.03393(03)$ & $1.61878(13)$ & $1.97517(05)$ & $2.17188(12)$ & $2.25759(06)$ \\
\hline-0.02 & $1.82633(04)$ & $2.09948(06)$ & $2.23256(10)$ & $2.26979(07)$ & $2.24376(10)$ \\
\hline-0.01 & $1.98098(11)$ & $2.18036(10)$ & $2.26182(12)$ & $2.26277(04)$ & $2.21200(10)$ \\
\hline 0.00 & $2.09535(15)$ & $2.23242(11)$ & $2.26953(32)$ & $2.24170(06)$ & $2.17054(13)$ \\
\hline 0.01 & $2.17596(08)$ & $2.26085(10)$ & $2.26303(13)$ & $2.20945(06)$ & $2.12214(12)$ \\
\hline 0.02 & $2.22885(15)$ & $2.27015(18)$ & $2.24196(04)$ & $2.16879(09)$ & $2.06838(06)$ \\
\hline 0.05 & $2.26502(06)$ & $2.21391(09)$ & $2.12432(10)$ & $2.01168(15)$ & $1.88826(06)$ \\
\hline$a$ & 2.0972 & 2.2339 & 2.2714 & 2.2425 & 2.1712 \\
\hline$b$ & 9.6367 & 3.9455 & -0.003 & -2.7032 & -4.5187 \\
\hline$c$ & -178.96 & -126.95 & -88.59 & -60.235 & -39.268 \\
\hline$d$ & 1069.7 & 802.33 & 597.81 & 440.48 & 330.16 \\
\hline
\end{tabular}

$\{20,30,40\}$ were used to calculate $\left\{T_{C}^{L^{\prime}=20}, T_{C}^{L^{\prime}=30}, T_{C}^{L^{\prime}=40}\right\}$. Thenceforth, the representative of the $T_{C}$ was estimated using the formulae [43]

$$
T_{C}=\left[\frac{T_{C}^{L=20}}{\left(\Delta T_{C}^{L=20}\right)^{2}}+\frac{T_{C}^{L=30}}{\left(\Delta T_{C}^{L=40}\right)^{2}}+\frac{T_{C}^{L=40}}{\left(\Delta T_{C}^{L=40}\right)^{2}}\right]\left(\Delta T_{C}\right)^{2},
$$

where

$$
\frac{1}{\left(\Delta T_{C}\right)^{2}}=\frac{1}{\left(\Delta T_{C}^{L=20}\right)^{2}}+\frac{1}{\left(\Delta T_{C}^{L=30}\right)^{2}}+\frac{1}{\left(\Delta T_{C}^{L=40}\right)^{2}},
$$

and $\Delta T_{C}$ is the statistical error of $T_{C}$. Results for the

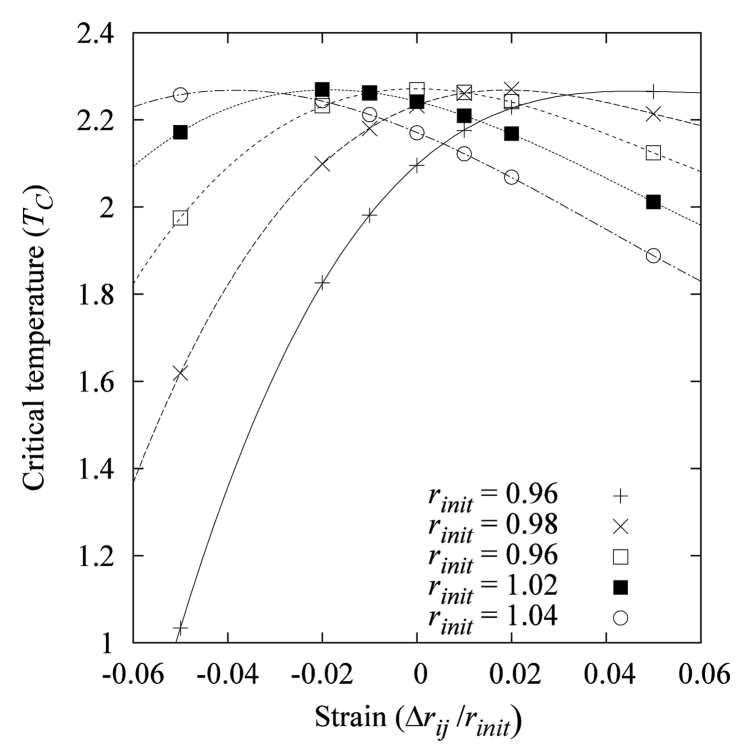

Fig. 4. The critical temperature results $T_{C}$ as a function of strain $\left(\Delta r_{i j} / r_{i n i t}\right)$ for various $r_{\text {init. }}$. The curves displayed are from the empirical polynomial degree-3 fit using the coefficients listed in Table 3. critical temperature $T_{C}$ for different values of $r_{\text {init }}$ and $\Delta r_{i j} /$ $r_{\text {init }}$ are given in Table 3 with the pictorial presentation in Fig. 4. In checking the accuracy of the results, the zero strain critical temperature was investigated, i.e. $T_{C}\left(r_{\text {init }}=\right.$ $\left.1.00, \Delta r_{i j} / r_{\text {init }}=0\right)=2.26953(32)$, which is in line with the exact analytic solution i.e. $k_{B} T_{C} \approx 2.2692 \quad$ [44] and previous applicable work [43]. This therefore confirms the reliability of the Monte Carlo calculation procedures used in this work.

In addition, as is seen in Fig. 4, the increase of the strain results in the increase or the decrease of the critical temperature depending on the condition of $r_{\text {init }}$ and $\Delta r_{i j} /$ $r_{\text {init }}$, which are obviously complicated in predicting. Therefore, scaling function based on empirical polynomial fitting was proposed on relating $T_{C}$ with $r_{\text {init }}$ and $\Delta r_{i j} / r_{\text {init }}$. By experimenting, the degree-3 polynomial (with $a, b, c$ and $b$ being the coefficients) i.e.

$$
T_{C}\left(x \equiv \frac{\Delta r_{i j}}{r_{0}}\right)=a+b x+c x^{2}+d x^{3}
$$

was found to work well for all $r_{\text {init }}$ conditions as the $r$ squared's are all larger than 0.9999. Results and qualities of the fit were shown in Fig. 4, where the coefficients in Eq. (10) for each $r_{\text {init }}$ were listed in Table 3. As a result, one can use these proposed scaling functions in estimating how the strain and the initial interatomic distance between nearest neighboring pairs of spins would affect the magnetic critical point in ultra-thin-film system.

It is also of interest to investigate the dynamic behavior of the ferromagnetic magnetic properties via the ferromagnetic hysteresis loops to observe the effect of stretchable spins. However, as it is well-known that not only the effect of $r_{\text {init }}$ and $\Delta r_{i j} / r_{\text {init }}$ but also the external field and temperature parameters influence the hysteresis behavior. 


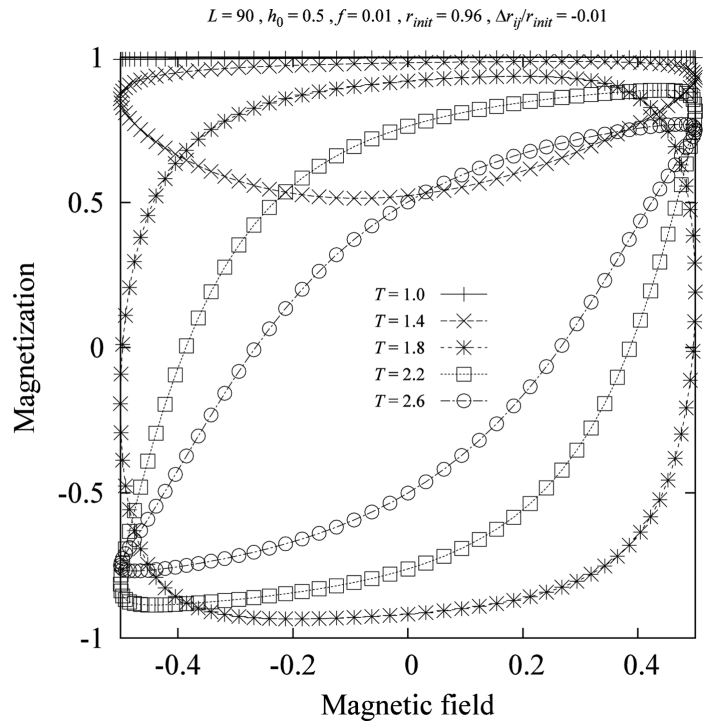

Fig. 5. The hysteresis loops of the two-dimensional stretchable Ising spins simulated with varying temperature $T$.

Therefore, the effect of temperature $T$, field amplitude $h_{0}$, the initial spacing $r_{\text {init }}$ and the strain $\Delta r_{i j} / r_{\text {init }}$ on hysteresis characteristic were investigated.

For instance, as can be seen in Fig. 5, the effect of temperature variation on the hysteresis loop was evident. The loops change from a straight line at $T=1.0 J_{0} / k_{B}$ to asymmetric hysteresis loop at $T=1.4 J_{0} / k_{B}$ and finally to a slim symmetric hysteresis loop at $T=2.6 J_{0} / k_{B}$. This is expected as higher temperatures bring larger thermal fluctuation into the system which compensates the ferromagnetic interaction among spins. Therefore, with larger fluctuation (at higher temperatures) the spins become less retarded to the magnetic field changing and so thus the phase-lag between the magnetization and the magnetic field signals. With smaller phase-lag, the hysteresis loops then change from asymmetric to symmetric characteristic [45].

On the other hand, with increasing the field amplitude, the system gains more magnetic driving force/works from the external field. This magnetic work indeed pulls the spins and the overall magnetization to change with the field. Therefore, as can be seen in Fig. 6, with large enough magnetic field amplitude, the energy supplied from field can help hysteresis loops changing from asymmetric hysteresis loop at $h_{0}=0.25 J_{0}$ to minor loop at $h_{0}=$ $0.50 J_{0}$ and finally to saturated symmetric loop at $h_{0}=$ $4.00 \mathrm{~J}_{0}$. The effect of field amplitude and temperature are in accordance with previous investigation on rigid Ising films $[45,46]$.

For the investigation of the effect of the initial spin-spin distance $r_{\text {init }}$ is its associated strain $\Delta r_{i j} / r_{\text {init }}$, interesting

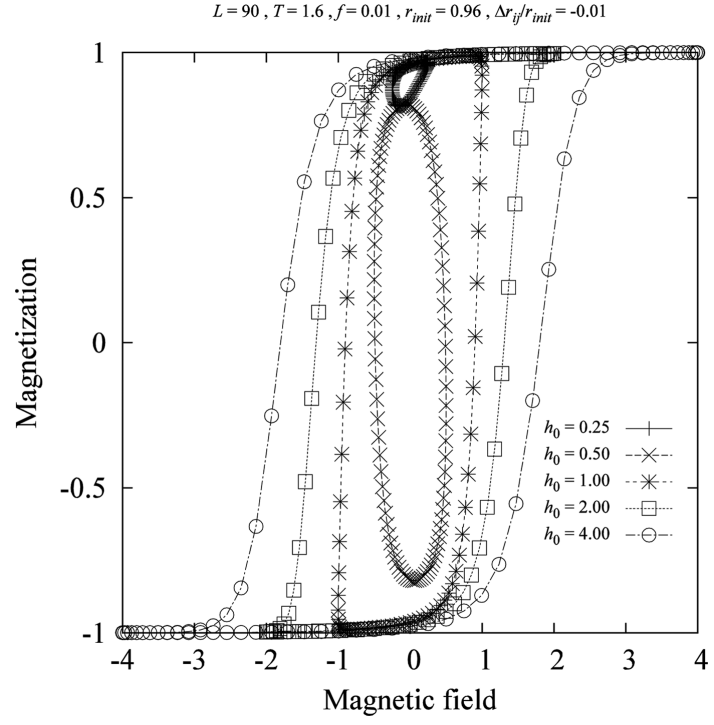

Fig. 6. The hysteresis loops of the two-dimensional stretchable Ising spins simulated with varying field amplitude $h_{0}$.

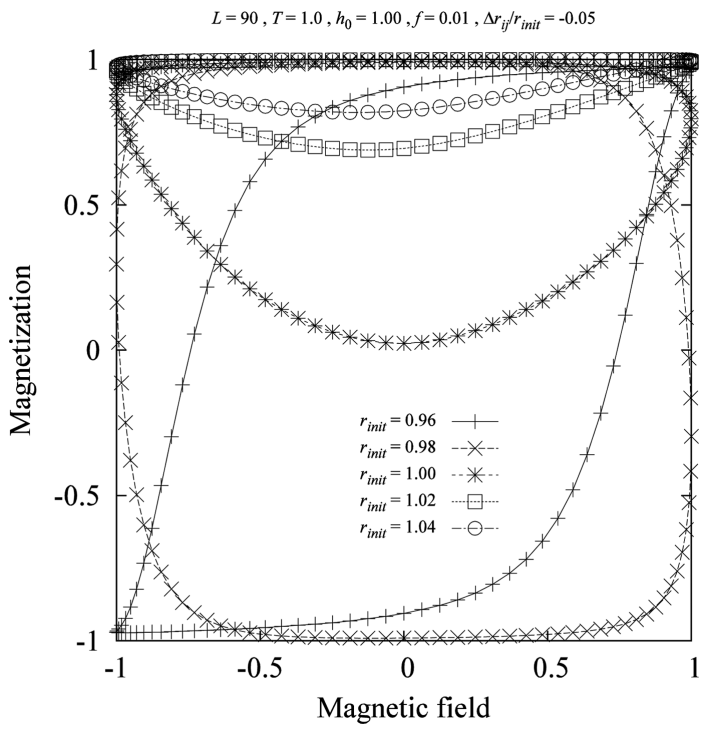

Fig. 7. The hysteresis loops of the two-dimensional stretchable Ising spins simulated with varying $r_{\text {init }}$.

results were found as can be shown in Figs. 7 and 8. For instance, with increasing the $r_{\text {init }}$, the symmetric hysteresis loop changes to asymmetric hysteresis loop as shown in Fig. 7. To describe this, the exchange interaction and the critical temperature results of the considered system have to be investigated in details. Considering the set of parameters used to simulate hysteresis loops in Fig. 7, both the exchange interaction $J_{i j}$ and the critical temperature $T_{C}$ enhances with increasing $r_{\text {init }}$ (at fixed $\Delta r_{i j} / r_{\text {init }}=-0.05$ ), e.g. see Tables 2 and 3. This indicates stronger ferromagnetic interaction among spins with increasing $r_{\text {init }}$, so this results in larger phase-lag inducing the hysteresis loop to 


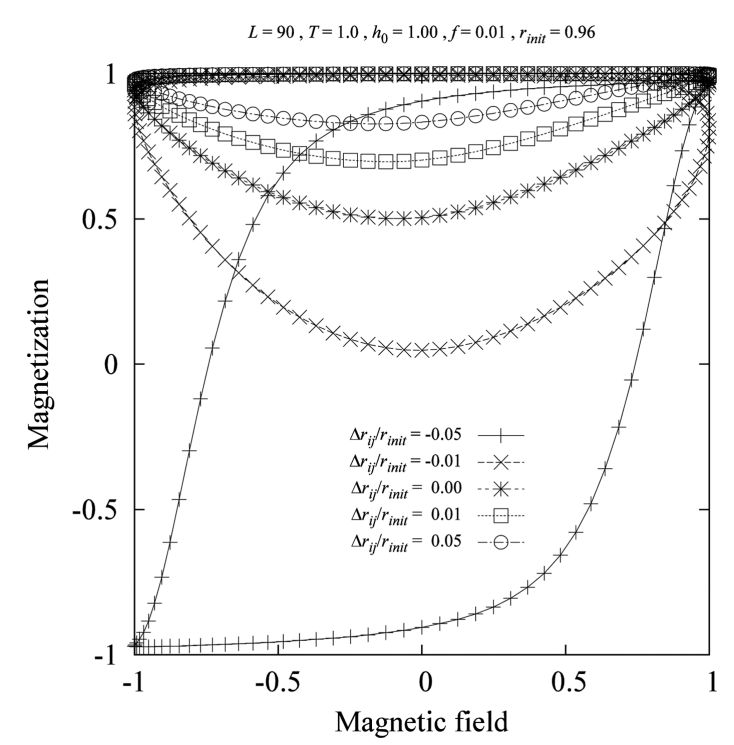

Fig. 8. The hysteresis loop of the two-dimensional stretchable Ising spins simulated with varying $\Delta r_{i j} / r_{\text {init }}$.

change from symmetric to asymmetric hysteresis loops with increasing $r_{\text {init. }}$. On the other hand, by keeping $r_{\text {init }}=$ 0.96 fixed but varying $\Delta r_{i j} / r_{\text {init }}$, the same description can be given for Fig. 8. At $r_{\text {init }}=0.96$, as indicated in Tables 2 and $3, J_{i j}, T_{C}$ and hence the ferromagnetic interaction enhance with increasing $\Delta r_{i j} / r_{\text {init }}$ from -0.05 to 0.05 . Therefore, the phase-lag advances which results in the increase in asymmetric behavior of the hysteresis loop with increasing with $\Delta r_{i j} / r_{\text {init }}$. This trend of hysteresis dependence of the ferromagnetic interaction corresponds with the increase of thickness or decrease of vacancy defect in Ising thin-films where the average ferromagnetic interaction among spins becomes larger [46, 47].

\section{Conclusion}

In summary, the work performed Monte Carlo simulations to investigate the effect the spatial variation between magnetic atoms to the initial interatomic distance ratio on the magnetic behavior of the two-dimensional Ising spins interacting via the Lennard-Jones type exchange interaction. The magnetic profiles were achieved while phase transition point was found to either increase or reduce depending on the value of both strain and the initial interatomic distance. The ferromagnetic-paramagnetic phase diagrams in presenting the critical conditions were established where the phase boundaries can be mathematically formulated using empirical polynomial fit. These critical temperature formulas can then be used to qualitatively provide how to control magnetic critical point in ultrathin-film using the substrate adjustment.

\section{References}

[1] A. Moser, K. Takano, D. T. Margulies, M. Albrecht, Y. Sonobe, Y. Ikeda, S. Sun, and E. E Fullerton, J. Phys. D: Appl. Phys. 35, R157 (2002).

[2] N. A. Spaldin, Magnetic Materials, Fundamentals and Device Applications, Cambridge University Press, Cambridge (2003).

[3] D. Sander, A. Enders, and J. Kirschner, J. Magn. Magn. Mater. 200, 439 (1999).

[4] W. Wulfhekel, F. Zavaliche, F. Porrati, H. P. Oepen, and J. Kirschner, Europhys. Lett. 49, 651 (2000).

[5] Y. Ohtani and I. Hatakeyama, J. Magn. Magn. Mater. 131, 339 (1994).

[6] T. Takikawa, T. Sakakibara, K. Matsuhira, K. Tenya, H. Amitsuka, and S. Kunii, Physica B 281, 561 (2000).

[7] A. Vl. Andrianov, E. Bauer, Ch. Paul, and O. A. Savel'eva, J. Magn. Magn. Mater. 272, E451 (2004).

[8] Y. V. Sushko, B. DeHarak, G. Cao, G. Shaw, D. K. Powell, and J. W. Brill, Solid State Commun. 130, 341 (2004).

[9] J. Prokop, D. A. Valdaitsev, A. Kukunin, M. Pratzer, G. Schönhense, and H. J. Elmers, Phys. Rev. B 70, 184423 (2004).

[10] R. Cheng, S. D. Bader, and F. Y. Fradin, Phys. Rev. B 77, 024404 (2008).

[11] C.-G. Duan, R. F. Sabiryanov, Jianjun Liu, W. N. Mei, P. A. Dowben, and J. R. Hardy, Phys. Rev. Lett. 94, 237201 (2005).

[12] T. Murata, H. Kushida, T. Terai, and T. Kakeshita, J. Magn. Magn. Mater. 310, 1555 (2007).

[13] B. Grossmann and D. G. Rancourt, Phys. Rev. B 54, 12294 (1996) and references therein.

[14] T. Burghardt, E. Hallmann, and A. Eichler, Physica B 230, 214 (1997).

[15] C. W. Looney, K. Falk, James J. Hamlin, Takahiro Tomita, James S. Schilling, W. Haase, and Z. Tomkowicz, Polyhedron 22, 3339 (2003).

[16] M. Nicklas, N. O. Moreno, H. A. Borges, E. D. Bauer, J. L. Sarrao, and J. D. Thompson, J. Magn. Magn. Mater. 272, E111 (2004).

[17] S. Arumugam, K. Mydeen, Magda Fontes, N. Manivannan, M. Kumaresa Vanji, K. U. RamaTulasi, S. M. Ramos, Elisa Baggio Saitovitch, D. Prabhakaran, and A. T. Boothroyd, Solid State Commun. 136, 292 (2005).

[18] V. A. Sidorov, A. V. Rakhmanina, and O. A. Morya, Solid State Commun. 139, 360 (2006).

[19] M. Mihalik, V. Kavečanský, A. S. Panfilov, K. Wochowski, and R. Troć, J. Alloys Compd. 421, 8 (2006).

[20] T. Hamasaki, H. Kuroe, T. Sekine, T. Naka, M. Hase, N. Maeshima, Y. Saiga, and Y. Uwatoko, J. Magn. Magn. Mater. 310, E394 (2007).

[21] C. S. Schneider, J. Appl. Phys. 97, 10E503 (2005).

[22] N.D. Mermin and H. Wagner, Phys. Rev. Lett. 17, 1133 (1966).

[23] P. C. Hohenberg, Phys. Rev. 158, 383 (1967). 
[24] D. Jansow and M. E. Fisher, Phys. Rev. Lett. 23, 286 (1969).

[25] P. A. Serena, N. García and A. Levanyuk, Phys. Rev. B 47, 5027 (1993).

[26] K. Binder and P. C. Hohenberg, Phys. Rev. B 9, 2194 (1974).

[27] M. Bander and D. L. Mills, Phys. Rev. B 38, 12015 (1988).

[28] Y. Li and K. Baberschke, Phys. Rev. Lett. 68, 1208 (1992).

[29] H. J. Elmers, J. Hauschild, H. Höche, U. Gradmann, H. Bethge, D. Heuer, and U. Köhler, Phys. Rev. Lett. 73, 898 (1994).

[30] M. J. Dunlavy and D. Venus, Phys. Rev. B 69, 094411 (2004).

[31] P. Massimino and H. T. Diep, J. Appl. Phys. 87, 7043 (2000).

[32] V. T. Ngo and H. T. Diep, J. Appl. Phys. 91, 8399 (2002).

[33] S. J. Mitchell and D. P. Landau, Phys. Rev. Lett. 97, 025701 (2006).

[34] R. C. Wayne and L. C. Bartel, Phys. Lett. A 28, 196 (1968).
[35] M. M. Abd-Elmeguid and H. Micklitz, Physica B 161, 17 (1989).

[36] J. M. D. Coey, Magnetism and Magnetic Materials, Cambridge University Press, Cambridge (2009).

[37] C. Heck, Magnetic Materials and Their Applications, Butterworth Co. (Publishers) Ltd., London (1974).

[38] U. Wolff, Phys. Rev. Lett. 62, 361 (1989).

[39] K. Binder and D. W. Heermann, Monte Carlo Simulation in Statistical Physics, Springer-Verlag, Berlin (1992).

[40] K. Binder, Z. Phys. B: Condens. Matter. 43, 119 (1981).

[41] M. E. J. Newman and G. T. Barkema, Monte Carlo Methods in Statistical Physics, Clarendon Press, Oxford (1999).

[42] N. Metropolis, A. W. Rosenbluth, M. N. Rosenbluth, A. H. Teller and E. Teller, J. Chem. Phys. 21, 1087 (1953).

[43] Y. Laosiritaworn, Ph.D. Thesis, The University of Warwick, UK (2002).

[44] B. M. McCoy and T. T. Wu, The Two-Dimensional Ising Model, Harvard University Press, Cambridge MA (1973).

[45] A. Punya, R. Yimnirun, P. Laoratanakul, and Y. Laosiritaworn, Physica B 405, 3482 (2010).

[46] Y. Laosiritaworn, Thin Solid Films 517, 5189 (2009).

[47] Y. Laosiritaworn, Adv. Mat. Res. 55, 385 (2008). 